\title{
STRICHARTZ ESTIMATES FOR THE SCHRÖDINGER EQUATION WITH A MEASURE-VALUED POTENTIAL
}

\author{
M. BURAK ERDOĞAN, MICHAEL GOLDBERG, AND WILLIAM R. GREEN
}

(Communicated by Alexander Iosevich)

\begin{abstract}
We prove Strichartz estimates for the Schrödinger equation in $\mathbb{R}^{n}$, $n \geqslant 3$, with a Hamiltonian $H=-\Delta+\mu$. The perturbation $\mu$ is a compactly supported measure in $\mathbb{R}^{n}$ with dimension $\alpha>n-\left(1+\frac{1}{n-1}\right)$. The main intermediate step is a local decay estimate in $L^{2}(\mu)$ for both the free and perturbed Schrödinger evolution.
\end{abstract}

\section{INTRODUCTION}

The dispersive properties of the free Schrödinger semigroup $e^{i t \Delta}$ are described in many ways, with one of the most versatile estimates being the family of Strichartz inequalities

$$
\left\|e^{i t \Delta} u\right\|_{L_{t}^{p} L_{x}^{q}} \lesssim\|u\|_{L^{2}\left(\mathbb{R}^{n}\right)}
$$

over the range $2 \leqslant p, q \leqslant \infty, \frac{2}{p}+\frac{n}{q}=\frac{n}{2}$, except for the endpoint $(p, q)=(2, \infty)$. There is a substantial body of literature devoted to establishing Strichartz inequalities and other dispersive bounds for the linear Schrödinger evolution of perturbed operators $H=-\Delta+V(x)$. [8, 15, 21] prove Strichartz inequalities for the free evolution. The latter two of these as well as [19] create a framework for extending them to perturbed Hamiltonians so long as the Schrödinger semigroup has suitable $L^{1} \rightarrow L^{\infty}$ dispersive bounds or $L^{2}\left(\mathbb{R}^{n} \times \mathbb{R}\right)$ smoothing. This strategy has been used to establish Strichartz estimates for the Schrödinger evolution for electric [13, magnetic [6] and time-periodic [9] perturbations. Most commonly $V(x)$ is assumed to exhibit pointwise polynomial decay or satisfying an integrability criterion such as belonging to a space $L_{l o c}^{r}\left(\mathbb{R}^{n}\right)$ for some $r \geqslant \frac{n}{2}$. Our goal in this paper is to show that Strichartz inequalities hold for a class of short-range potentials $V(x)$ that include measures $\mu(d x)$ as admissible local singularities.

Measure-valued potentials are often considered in one dimension; the operator $-\frac{d^{2}}{d x^{2}}+c \delta_{0}$ is often the subject of exercises in an introductory quantum mechanics course. In higher dimensions there are several plausible generalizations of this example. The three-dimensional Schrödinger operator $H=-\Delta+\sum c_{j} \delta\left(x_{j}\right)$ is studied in [3] and [4, and in two dimensions in [2]. Here, the singularity of the potential imposes boundary conditions at each point $x_{j}$ for functions belonging to

Received by the editors August 7, 2019.

2020 Mathematics Subject Classification. Primary 35Q40; Secondary 42B15.

The first author was partially supported by NSF grant DMS-1501041.

The second author was supported by Simons Foundation Grant 281057.

The third author was supported by Simons Foundation Grant 511825.

(C) 2021 by the authors under Creative Commons Attribution-Noncommercial 3.0 License (CC BY NC 3.0) 
the domain of $H$. As an eventual consequence, linear dispersive and Strichartz inequalities hold only on a subset of the range described above.

The potentials considered in this paper are less singular than a delta-function in $\mathbb{R}^{n}$, but still not absolutely continuous with respect to Lebesgue measure. The surface measure of a compact hypersurface $\Sigma \subset \mathbb{R}^{n}$ is a canonical example of an admissible potential we consider. More generally we work with compactly supported fractal measures (on $\mathbb{R}^{n}$ ) of a sufficiently high dimension. The exact threshold will be determined in context. Arguments regarding the self-adjointness of $H$ require a dimension greater than $n-2$ so that multiplication by $\mu$ remains compact relative to the Laplacian. We are forced to increase the threshold dimension to $n-\left(1+\frac{1}{n-1}\right)$ in the proof of the local decay and Strichartz estimates. Under these conditions, and a modest assumption about the spectral properties of $H$, we prove that the entire family of Strichartz inequalities (1.1) is preserved with the possible exception of the $(p, q)=\left(2, \frac{2 n}{n-2}\right)$ endpoint.

With $B(x, r)$ a ball of radius $r$ centered at $x \in \mathbb{R}^{n}$, we say that a compactly supported signed measure $\mu$ is $\alpha$-dimensional if it satisfies

$$
|\mu|(B(x, r)) \leqslant C_{\mu} r^{\alpha} \text { for all } r>0 \text { and } x \in \mathbb{R}^{n} .
$$

Nontrivial $\alpha$-dimensional measures exist for any $\alpha \in[0, n]$.

The first obstruction to Strichartz estimates with a Hamiltonian $H=-\Delta+V$ is the possible existence of bound states, functions $\psi \in L^{2}\left(\mathbb{R}^{n}\right)$ that solve $H \psi=E \psi$ for some real number $E$. Each bound state gives rise to a solution of the Schrödinger equation $e^{i t H} \psi(x)=e^{i t E} \psi(x)$, which satisfies (1.1) only for $(p, q)=(\infty, 2)$ and no other choice of exponents.

Our main result asserts that the perturbed evolution $e^{i t H}$ satisfies Strichartz estimates once all bound states of $H$ are projected away. We impose additional spectral assumptions that all eigenvalues of $H$ are strictly negative, and that there is no resonance at zero. In this paper we say a resonance occurs at $\lambda$ when the equation

$$
\psi+(-\Delta-(\lambda \pm i 0))^{-1} \mu \psi=0
$$

has nontrivial solutions belonging to the Sobolev space $\dot{H}^{1}\left(\mathbb{R}^{n}\right)$ but not to $L^{2}$ itself.

Thanks to the compact support of $\mu$, one can easily show that resonances are impossible at $\lambda<0$, and can only occur at $\lambda=0$ in dimensions 3 and 4 . We show in Section 3 that resonances do not occur when $\lambda>0$. Eigenvalues at zero are possible provided the negative part of the potential is large enough. Positive eigenvalues are known to be absent for a wide class of potentials (see [16]) covering some (but not all) of the measures considered here; see Remark 3.2 .

To state our results, we define the following $L^{p}$ spaces. For $\mu$ a signed measure on $\mathbb{R}^{n}$, we define

$$
L^{p}(\mu):=\left\{f: \mathbb{R}^{n} \rightarrow \mathbb{C}: \int_{\mathbb{R}^{n}}|f|^{p} d|\mu|<\infty\right\}
$$

for $1 \leqslant p<\infty$. With the natural, minor modification one can define $L^{\infty}(\mu)$. It is worthwhile to note that multiplication by $\mu$ is an isometry from $L^{p}(\mu)$ to $L^{p^{\prime}}(\mu)^{*}$ with $p^{\prime}$ the Hölder conjugate of $p$ for any $1 \leqslant p \leqslant \infty$. This can be seen easily by using the natural duality pairing. Throughout the paper we will take particular advantage of the fact that multiplication by $\mu$ maps $L^{2}(\mu)$ to its dual space. Finally, let $P_{a c}$ denotes projection onto the continuous spectrum of $-\Delta+\mu$. 
Theorem 1.1. Let $\mu$ be a compactly supported signed measure on $\mathbb{R}^{n}$ of dimension $\alpha>n-\left(1+\frac{1}{n-1}\right)$. If the Schrödinger operator $-\Delta+\mu$ has no resonance at zero and no eigenvalues at any $\lambda \geqslant 0$, then for each $f \in L^{2}\left(\mathbb{R}^{n}\right)$ we have the local decay bounds

$$
\begin{aligned}
\left\|e^{i t \Delta} f\right\|_{L_{t}^{2} L^{2}(\mu)} & \lesssim\|f\|_{2}, \\
\left\|e^{i t(-\Delta+\mu)} P_{a c} f\right\|_{L_{t}^{2} L^{2}(\mu)} & \lesssim\|f\|_{2}
\end{aligned}
$$

and the Strichartz inequalities

$$
\left\|e^{i t(-\Delta+\mu)} P_{a c} f\right\|_{L_{t}^{p} L_{x}^{q}} \lesssim\|f\|_{2}
$$

for admissible pairs $(p, q)$ with $\frac{2}{p}+\frac{n}{q}=\frac{n}{2}$ and $p>2$.

The second author considered $L^{1} \rightarrow L^{\infty}$ dispersive estimates in $\mathbb{R}^{3}$ (under the same set of assumptions when $n=3$, including $\alpha>\frac{3}{2}$ ) in [10]. Strichartz inequalities in this case follow as a direct consequence by [15. The results presented here in $\mathbb{R}^{n}, n \geqslant 4$, are new and rely in part on recent advances in Fourier restriction problems such as [5,17]. In particular the improved decay of spherical Fourier means allows us to capture the physically relevant $\alpha=n-1$ case where the potential might be supported on a compact hypersurface in higher dimensions. It is known for dimensions $n>3$ that $L^{1} \rightarrow L^{\infty}$ dispersive estimates need not hold even for compactly supported potentials $V$ if $V$ is not sufficiently differentiable; see [7,12. Whereas the smoothness is not required for Strichartz estimates to hold in higher dimensions. Our argument works in dimensions $n \geqslant 3$; technical issues in dimension $n=2$ (for example with the use of $\dot{H}^{1}\left(\mathbb{R}^{n}\right)$ ) would require different methods.

$\alpha$-Dimensional measures also satisfy a strong Kato-type property that for any $\gamma<\alpha$,

$$
\sup _{y \in \mathbb{R}^{n}} \int_{|x-y|<r} \frac{|\mu|(d x)}{|x-y|^{\gamma}} \lesssim C_{\mu} r^{\alpha-\gamma} .
$$

Furthermore, since $\mu$ has compact support, the integral over the entire space $x \in \mathbb{R}^{n}$ is bounded uniformly in $y$. These integral bounds will be proved as Lemma 2.2. The choice $\gamma=n-2$ is significant due to its connection with the Green's function of the Laplacian in $\mathbb{R}^{n}$ when $n \geqslant 3$.

We also characterize potentials in terms of the global Kato norm, defined on signed measures in $\mathbb{R}^{n}$ by the quantity

$$
\|\mu\|_{\mathcal{K}}=\sup _{y \in \mathbb{R}^{n}} \int_{\mathbb{R}^{n}} \frac{|\mu|(d x)}{|x-y|^{n-2}} .
$$

One can see that every element with finite global Kato norm is an $(n-2)$-dimensional measure with $C_{\mu} \leqslant\|\mu\|_{\mathcal{K}}$, by comparing $|x-y|^{2-n}$ to the characteristic function of a ball. The converse is false; however the Kato class contains all compactly supported measures of dimension $\alpha>n-2$. We examine this relationship in Lemma 2.2. We follow the naming convention in Rodnianski-Schlag [19] where the global Kato norm is applied to dispersive estimates in $\mathbb{R}^{3}$, as opposed to the local norms considered in Schechter [20].

There is a now well-known strategy to obtain the Strichartz estimates (1.6). One uses the space-time $L^{2}$ estimates (1.4) and (1.5) and the argument of RodnianskiSchlag [19]. There is a minor modification to the Rodnianski-Schlag framework in 
that instead of factorizing the operator corresponding to multiplication by $\mu$, we instead apply it directly as a bounded map from $L_{t}^{2} L^{2}(\mu)$ to its dual space.

The resolvent operators $(-\Delta-\lambda)^{-1}$ and $(-\Delta+\mu-\lambda)^{-1}$ are well defined for $\lambda$ in the resolvent sets. We define the limiting resolvent operators

$$
R_{0}^{ \pm}(\lambda):=\lim _{\varepsilon \rightarrow 0^{+}}(-\Delta-(\lambda \pm i \varepsilon))^{-1} \quad \text { and } \quad R_{\mu}^{ \pm}(\lambda):=\lim _{\varepsilon \rightarrow 0^{+}}(-\Delta+\mu-(\lambda \pm i \varepsilon))^{-1} .
$$

Following Kato's derivation [14, $L^{2}$ estimates such as (1.4) and (1.5) are valid precisely if there are uniform bounds of the resolvent operators. We prove the following mapping bounds for the resolvents $R_{0}^{ \pm}(\lambda)$ and $R_{\mu}^{ \pm}(\lambda)$.

Theorem 1.2. Under the hypotheses of Theorem 1.1

$$
\begin{array}{ll} 
& \sup _{\lambda \geqslant 0} \|\left(\left(R_{0}^{+}(\lambda)-R_{0}^{-}(\lambda)\right) \mu \|_{L^{2}(\mu) \rightarrow L^{2}(\mu)}<\infty\right. \\
\text { and } & \sup _{\lambda \geqslant 0} \|\left(\left(R_{\mu}^{+}(\lambda)-R_{\mu}^{-}(\lambda)\right) \mu \|_{L^{2}(\mu) \rightarrow L^{2}(\mu)}<\infty .\right.
\end{array}
$$

Due to the different challenges of establishing these bounds when the spectral parameter is close to $\lambda=0$ (small energy) or bounded away from zero (large energy), we require different tools in each regime. We bound the low energy contribution in Section 3 in Lemma 3.1. while the large energy is controlled in Section 4 Once the resolvent bounds are established at all energies, we assemble the results to prove Theorem 1.1

\section{SElF-ADJOINTNESS AND COMPACTNESS}

For any perturbation $V(x)$ which is not a bounded function of $x$ there are well known difficulties identifying the domain of $-\Delta+V$ and its adjoint operator. The main goal of this section is to prove Proposition 2.1. Along the way, we will prove some compactness results that will be useful for describing the spectral measure of $-\Delta+\mu$.

Proposition 2.1. If $\mu$ is a compactly supported $\alpha$-dimensional signed measure for some $\alpha>n-2$, then there exists a unique self-adjoint extension of $-\Delta+\mu$.

The first step is to check that $\mu$ satisfies both a local and global "Kato condition."

Lemma 2.2. Suppose $\mu$ is an $\alpha$-dimensional signed measure with support in the ball $B(0, M)$, and $\gamma$ is such that $\alpha>\gamma>0$. Then $\mu$ satisfies the estimates

$$
\begin{aligned}
& \sup _{y \in \mathbb{R}^{n}} \int_{|x-y|<r} \frac{|\mu|(d x)}{|x-y|^{\gamma}} \lesssim C_{\mu} r^{\alpha-\gamma} \quad \text { for all } r>0 \\
& \text { and } \sup _{y \in \mathbb{R}^{n}} \int_{\mathbb{R}^{n}} \frac{|\mu|(d x)}{|x-y|^{\gamma}} \lesssim C_{\mu} M^{\alpha-\gamma} .
\end{aligned}
$$

Proof. For each point $y \in \mathbb{R}^{n}$,

$$
\begin{aligned}
\int_{|x-y|<r} \frac{|\mu|(d x)}{|x-y|^{\gamma}} & =\gamma \int_{0}^{r} \frac{|\mu|(B(y, t))}{t^{\gamma+1}} d t+r^{-\gamma}|\mu|(B(y, r)) \\
& \lesssim C_{\mu}\left(\int_{0}^{r} t^{\alpha-\gamma-1} d t+r^{\alpha-\gamma}\right)=C_{\mu}\left(1+\frac{1}{\alpha-\gamma}\right) r^{\alpha-\gamma}
\end{aligned}
$$

This establishes the first claim. For the second claim, if $|y|<2 M$, the global bound is achieved by setting $r=3 M$. If $|y|>2 M$ then the integral in (2.1) is 
easily bounded by $|y|^{-\gamma}|\mu|(B(0, M))$ by observing that $|x-y| \sim|y| \gtrsim M$ within the support of $\mu$.

By choosing $\gamma=n-2$, it follows that $\|\mu\|_{\mathcal{K}}<\infty$.

Lemma 2.3. If $\mu$ is a compactly supported $\alpha$-dimensional measure for some $\alpha>$ $n-2$, then $\dot{H}^{1}\left(\mathbb{R}^{n}\right) \subset L^{2}(\mu)$.

Proof. Two mapping bounds follow directly from the definition of the global Kato norm, using that the integral kernel of $(-\Delta)^{-1}(x, y)$ is a scalar multiple of $|x-y|^{2-n}$,

$$
\begin{aligned}
\left\|(-\Delta)^{-1} \mu f\right\|_{L^{\infty}(\mu)} & \lesssim\|\mu\|_{\mathcal{K}}\|f\|_{L^{\infty}(\mu)}, \\
\left\|(-\Delta)^{-1} \mu f\right\|_{L^{1}(\mu)} & \lesssim\|\mu\|_{\mathcal{K}}\|f\|_{L^{1}(\mu)} .
\end{aligned}
$$

Interpolation between these two endpoints yields

$$
\left\|(-\Delta)^{-1} \mu f\right\|_{L^{2}(\mu)} \lesssim\|\mu\|_{\mathcal{K}}\|f\|_{L^{2}(\mu)} .
$$

This along with a $T T^{*}$ argument shows that the square root $(-\Delta)^{-\frac{1}{2}}$ is a bounded operator from $L^{2}\left(\mathbb{R}^{n}\right)$ to $L^{2}(\mu)$; by duality it is also bounded from $L^{2}(\mu)^{*}$ to $L^{2}\left(\mathbb{R}^{n}\right)$. At the same time $(-\Delta)^{-\frac{1}{2}}$ is an isometry from $L^{2}\left(\mathbb{R}^{n}\right)$ onto $\dot{H}^{1}\left(\mathbb{R}^{n}\right)$. This suffices to prove the desired inclusion. Further, (2.2) shows that

$$
\left\|(-\Delta)^{-1} g\right\|_{L^{2}(\mu)} \lesssim\|\mu\|_{\mathcal{K}}\|g\|_{L^{2}(\mu) *} .
$$

Given a point $z \in \mathbb{R}^{n}$, define the translation operator $\tau_{z} f(x):=f(x-z)$. Translation operators are not bounded on $L^{2}(\mu)$ in general, but they behave quite well when restricted to the subspace $\dot{H}^{1}$. Let $j: \dot{H}^{1}\left(\mathbb{R}^{n}\right) \rightarrow L^{2}(\mu)$ be the natural inclusion operator.

Lemma 2.4. If $\mu$ is a compactly supported $\alpha$-dimensional measure for some $\alpha>$ $n-2$,

$$
\left\|\left(j-\tau_{z}\right) \varphi\right\|_{L^{2}(\mu)} \lesssim \sqrt{C_{\mu}}|z|^{\beta}\|\varphi\|_{\dot{H}^{1}}
$$

for any $0<\beta<\frac{\alpha-(n-2)}{2}$ and $|z|<1$.

Proof. By a $T T^{*}$ argument, it suffices to show that

$$
\left\|\left(j-\tau_{z}\right)(-\Delta)^{-1}\left(j^{*}-\tau_{-z}\right) g\right\|_{L^{2}(\mu)} \lesssim C_{\mu}|z|^{2 \beta}\|g\|_{L^{2}(\mu) *},
$$

where $j^{*}$ is the inclusion of $L^{2}(\mu)^{*}$ into $\dot{H}^{-1}$. Translations commute with powers of the Laplacian, so there is another equivalent statement

$$
\left\|\left(2-\tau_{z}-\tau_{-z}\right)(-\Delta)^{-1} \mu f\right\|_{L^{2}(\mu)} \lesssim C_{\mu}|z|^{2 \beta}\|f\|_{L^{2}(\mu)} .
$$

Here we use that $j$ and $j^{*}$ may be replaced with the operators $\tau_{0}$ or 1 on their respective domains. We now show that

$$
\left|\int_{\mathbb{R}^{n}}\left(\frac{2}{|x-y|^{n-2}}-\frac{1}{|x-(y-z)|^{n-2}}-\frac{1}{|x-(y+z)|^{n-2}}\right) \mu(d x)\right| \lesssim C_{\mu}|z|^{2 \beta} .
$$

Indeed, Lemma 2.2 immediately proves this bound for the integral over the region where $|x-y| \leqslant 2|z|^{2 \beta /(\alpha-n+2)}$. Since the exponent $2 \beta /(\alpha-n+2)$ is strictly less than 1 , the region includes all three singularities at $x \in\{y, y-z, y+z\}$. 
Outside of the region, Taylor's remainder theorem controls the integrand by a multiple of $\frac{|z|^{2}}{|x-y|^{n}}$. We write

$$
\frac{1}{|x-y|^{n}}=\frac{1}{|x-y|^{\frac{\alpha-n+2}{\beta}+n-\alpha-2}} \frac{1}{|x-y|^{\alpha+2-\frac{\alpha-n+2}{\beta}}},
$$

and note that under the hypotheses, both exponents are positive. On the region of interest, the first term is dominated by $|z|^{2 \beta-2}$. The estimate continues with

$$
\begin{aligned}
\int_{|x-y|>|z|^{\frac{2 \beta}{\alpha-n+2}}} \frac{|z|^{2}}{|x-y|^{n}}|\mu|(d x) & \lesssim \int_{\mathbb{R}^{n}} \frac{|z|^{2}}{|z|^{2-2 \beta}|x-y|^{\alpha+2-\frac{a-n+2}{\beta}}}|\mu|(d x) \\
& =\int_{\mathbb{R}^{n}} \frac{|z|^{2 \beta}}{|x-y|^{\alpha+2-\frac{\alpha-n+2}{\beta}}}|\mu|(d x) \lesssim C_{\mu}|z|^{2 \beta} .
\end{aligned}
$$

The last inequality follows from Lemma 2.2 since $\frac{\alpha-n+2}{\beta}>2$, making the exponent in the denominator less than $\alpha$.

The bound in (2.4) implies that

$$
\begin{aligned}
\left\|\left(2-\tau_{z}-\tau_{-z}\right)(-\Delta)^{-1} \mu f\right\|_{L^{\infty}(\mu)} & \lesssim C_{\mu}|z|^{2 \beta}\|f\|_{L^{\infty}(\mu)}, \\
\left\|\left(2-\tau_{z}-\tau_{-z}\right)(-\Delta)^{-1} \mu f\right\|_{L^{1}(\mu)} & \lesssim C_{\mu}|z|^{2 \beta}\|f\|_{L^{1}(\mu)} .
\end{aligned}
$$

The desired $L^{2}(\mu)$ bound follows by interpolation.

Lemma 2.5. The embedding $j: \dot{H}^{1}\left(\mathbb{R}^{n}\right) \rightarrow L^{2}(\mu)$ is compact.

Proof. Let $\eta_{r}$ be a standard mollifier supported in a ball of radius $0<r<1$. Lemma 2.4 implies that

$$
\left\|\varphi-\left(\eta_{r} * \varphi\right)\right\|_{L^{2}(\mu)} \lesssim r^{\beta}\|\varphi\|_{\dot{H}^{1}} .
$$

Furthermore, if $\chi$ is any smooth cutoff that is identically 1 on the support of $\mu$, then multiplication by $\chi$ has no effect in $L^{2}(\mu)$. Thus

$$
\left\|\varphi-\left(\eta_{r} * \varphi\right) \chi\right\|_{L^{2}(\mu)} \lesssim r^{\beta}\|\varphi\|_{\dot{H}^{1}} .
$$

The operators mapping $\varphi$ to $\left(\eta_{r} * \varphi\right) \chi$ are compact on $\dot{H}^{1}\left(\mathbb{R}^{n}\right)$, so they are also compact from $\dot{H}^{1}$ to $L^{2}(\mu)$. We have just shown that they converge to the inclusion map $j$ as $r \rightarrow 0$.

Corollary 2.6. For any fixed $\lambda \geqslant 0$, the operator $R_{0}^{+}\left(\lambda^{2}\right) \mu$ is compact on $L^{2}(\mu)$.

Proof. Recall that $R_{0}(0)$ is the same as $(-\Delta)^{-1}$ in dimensions $n \geqslant 3$. Then $R_{0}(0) \mu$ is the composition $j(-\Delta)^{-1} j^{*} \mu$, with both inclusions $j$ and $j^{*}$ being compact.

For $\lambda>0$, the free resolvents $R_{0}^{+}\left(\lambda^{2}\right)$ map weighted $\dot{H}^{-1}\left(\mathbb{R}^{n}\right)$ into weighted $\dot{H}^{1}\left(\mathbb{R}^{n}\right)$. Then, with $\chi$ again a smooth cutoff to the support of $\mu, \chi R_{0}^{+}\left(\lambda^{2}\right) \chi$ is a bounded map from $\dot{H}^{-1}$ to $\dot{H}^{1}$ without additional weights due to the compact support of $\mu$. We may write

$$
R_{0}^{+}\left(\lambda^{2}\right) \mu=j\left(\chi R_{0}^{+}\left(\lambda^{2}\right) \chi\right) j^{*} \mu
$$

with $j$ and $j^{*}$ once again being compact.

Remark 2.7. The derivative $\chi \frac{d}{d \lambda} R_{0}^{+}\left(\lambda^{2}\right) \chi$ is also a bounded map from $\dot{H}^{-1}$ to $\dot{H}^{1}$. The same argument as above shows that the family of operators $R_{0}^{+}\left(\lambda^{2}\right) \mu: L^{2}(\mu) \rightarrow$ $L^{2}(\mu)$ are differentiable with respect to $\lambda$. 
Proof of Proposition 2.1. We can take advantage of the KLMN theorem 18, Theorem X.17] to produce a unique self-adjoint operator with the correct quadratic form on $\dot{H}^{1}\left(\mathbb{R}^{n}\right)$ provided $\mu$ satisfies the form bound

$$
\left.\left|\int_{\mathbb{R}^{n}}\right| \varphi(x)\right|^{2} d \mu \mid \leqslant a\|\varphi\|_{\dot{H}^{1}}^{2}+b\|\varphi\|_{L^{2}}^{2}
$$

for some $a<1$. For $|z|<1$ and $\varphi \in \dot{H}^{1}\left(\mathbb{R}^{n}\right)$, by Lemma 2.4 we have

$$
\begin{gathered}
\int_{\mathbb{R}^{n}}|\varphi(x)|^{2} d \mu-\int_{\mathbb{R}^{n}}|\varphi(x)|^{2} d\left(\tau_{z} \mu\right)=\int_{\mathbb{R}^{n}}\left(|\varphi(x)|^{2}-|\varphi(x+z)|^{2}\right) d \mu \\
=\int_{\mathbb{R}^{n}}(\varphi(x)-\varphi(x+z)) \bar{\varphi}(x) d \mu+\int_{\mathbb{R}^{n}} \varphi(x+z)(\bar{\varphi}(x)-\bar{\varphi}(x+z)) d \mu \\
\leqslant\left\|\left(j-\tau_{-z}\right) \varphi\right\|_{L^{2}(\mu)}\left(\|\varphi\|_{L^{2}(\mu)}+\left\|\tau_{-z} \varphi\right\|_{L^{2}(\mu)}\right) \lesssim|z|^{\beta}\|\varphi\|_{\dot{H}^{1}}^{2} .
\end{gathered}
$$

It follows that

$$
\left.\left|\int_{\mathbb{R}^{n}}\right| \varphi(x)\right|^{2} d \mu-\left.\int_{\mathbb{R}^{n}}|\varphi(x)|^{2}\left(\mu * \eta_{r}\right)(x) d x|\lesssim| r\right|^{\beta}\|\varphi\|_{\dot{H}^{1}}^{2}
$$

for a mollifier $\eta_{r}$ supported in a ball radius $r$. At the same time $\mu * \eta_{r}$ is a bounded function for each $r>0$, so there is a second estimate

$$
\left.\left|\int_{\mathbb{R}^{n}}\right| \varphi(x)\right|^{2}\left(\mu * \eta_{r}\right)(x) d x \mid \leqslant C_{r}\|\varphi\|_{L^{2}\left(\mathbb{R}^{n}\right)}^{2} .
$$

Allowing $r$ to approach zero shows that (2.5) holds with any choice of $a>0$.

Proposition 2.8. If $\mu$ is a compactly supported $\alpha$-dimensional signed measure for some $\alpha>n-2$, then $-\Delta+\mu$ has finitely many negative eigenvalues and no other spectrum in $(-\infty, 0)$.

Proof. Since (2.5) holds for some $0<a<1$, the operator $-\Delta+\mu$ is bounded from below. Then the range of the spectral projection $P_{(-\infty, 0)}$ is a closed subspace of $L^{2}$ contained inside the negative-definite subspace of the quadratic form $((-\Delta+$ $\mu) \varphi, \varphi)$. On this subspace we also have the bound

$$
\|\varphi\|_{\dot{H}^{1}}^{2} \leqslant((-\Delta+\mu) \varphi, \varphi)+a\|\varphi\|_{\dot{H}^{1}}^{2}+b\|\varphi\|_{L^{2}}^{2} \leqslant a\|\varphi\|_{\dot{H}^{1}}^{2}+b\|\varphi\|_{L^{2}}^{2}
$$

and it follows that $\|\varphi\|_{\dot{H}^{1}} \lesssim\|\varphi\|_{L^{2}}$.

Consider the factorization

$$
-\Delta+\mu=(-\Delta)^{1 / 2}\left[I+(-\Delta)^{-1 / 2} \mu(-\Delta)^{-1 / 2}\right](-\Delta)^{1 / 2} .
$$

The central operator is a compact and self-adjoint perturbation of the identity acting on $L^{2}\left(\mathbb{R}^{n}\right)$, namely $I+(-\Delta)^{-1 / 2} j^{*} \mu j(-\Delta)^{-1 / 2}$. Its negative-definite subspace is finite dimensional. As observed above, the range of $P_{(-\infty, 0)}$ is contained in $L^{2}\left(\mathbb{R}^{n}\right) \cap \dot{H}^{1}\left(\mathbb{R}^{n}\right)$. The square-root of the Laplacian maps this space into $L^{2}$ in a one-to-one manner. Thus the range of $P_{(-\infty, 0)}$ is also finite dimensional, with dimension no larger than the negative-definite space of $I+(-\Delta)^{-1 / 2} \mu(-\Delta)^{-1 / 2}$.

\section{LOW ENERGY ESTIMATES}

At this point we establish a uniform bound on the low energy perturbed resolvent as an operator on $L^{2}(\mu)$. Specifically, we show 
Lemma 3.1. Let $\mu$ be a real-valued measure on $\mathbb{R}^{n}, n \geqslant 3$, with dimension $\alpha>$ $n-2$. If $(-\Delta+\mu)$ has no eigenvalues at $\lambda \geqslant 0$, and (if $n=3,4)$ no resonances at $\lambda=0$, then

$$
\sup _{|\lambda| \leqslant L}\left\|R_{\mu}^{+}\left(\lambda^{2}\right) \mu\right\|_{L^{2}(\mu) \rightarrow L^{2}(\mu)} \leqslant C_{L}<\infty
$$

for any $L>0$, with a fixed constant that depends on $L$.

Proof. The estimation of perturbed resolvents on a finite interval follows a standard procedure. First, we express the perturbed resolvent $R_{\mu}^{+}\left(\lambda^{2}\right) \mu$ using the identity

$$
R_{\mu}^{+}\left(\lambda^{2}\right) \mu=\left(I+R_{0}^{+}\left(\lambda^{2}\right) \mu\right)^{-1} R_{0}^{+}\left(\lambda^{2}\right) \mu .
$$

The operators $R_{0}^{+}\left(\lambda^{2}\right) \mu: L^{2}(\mu) \rightarrow L^{2}(\mu)$ are continuous with respect to $\lambda$, so they are uniformly bounded over any finite interval. If an inverse $\left(I+R_{0}^{+}\left(\lambda^{2}\right) \mu\right)^{-1}$ exists at each $\lambda \geqslant 0$, then the inverses will be continuous, and uniformly bounded on each finite interval.

Suppose $I+R_{0}^{+}\left(\lambda_{0}^{2}\right) \mu$ fails to be invertible on $L^{2}(\mu)$ for some $\lambda_{0}>0$. By the Fredholm alternative, there must exist a nontrivial $\psi \in L^{2}(\mu)$ belonging to its null space. This function satisfies the bootstrapping relation

$$
\psi=-R_{0}^{+}\left(\lambda_{0}^{2}\right) \mu \psi
$$

Assuming $\mu$ is real-valued, the duality pairing $(\mu \psi, \psi)=\int_{\mathbb{R}^{n}}\left|\psi^{2}(x)\right| d \mu$ is realvalued as well. It is also equal to the pairing

$$
-\left(\mu \psi, R_{0}^{+}\left(\lambda_{0}^{2}\right) \mu \psi\right)=\int_{\mathbb{R}^{n}} \frac{|\widehat{\mu \psi}(\xi)|^{2}}{|\xi|^{2}-\left(\lambda_{0}+i 0\right)^{2}} d \xi
$$

whose imaginary part is a multiple of $\int_{\left\{|\xi|=\lambda_{0}\right\}}|\widehat{\mu \psi}(\xi)|^{2}$. In order for this quantity to be real, the Fourier transform of $\mu \psi$ must vanish on the sphere of radius $\lambda_{0}$.

Let $\eta$ be a mollifier whose Fourier transform is identically 1 when $|\xi| \leqslant 2 \lambda_{0}$. Convolution against $\eta$ is a bounded operator on $\dot{H}^{-1}$ and it maps finite measures on $\mathbb{R}^{n}$ to $L^{p}\left(\mathbb{R}^{n}\right), 1 \leqslant p \leqslant \infty$. In particular, $\eta * \mu \psi \in L^{\frac{2 n+2}{n+5}}\left(\mathbb{R}^{n}\right)$, along with the fact that its Fourier transform vanishes on the sphere of radius $\lambda_{0}$; it follows from [11, Theorem 2] that $R_{0}^{+}\left(\lambda_{0}^{2}\right)(\eta * \mu \psi) \in L^{2}\left(\mathbb{R}^{n}\right)$.

Meanwhile $\mu \psi-(\eta * \mu \psi) \in \dot{H}^{-1}\left(\mathbb{R}^{n}\right)$, and it has Fourier support where $|\xi|>2 \lambda_{0}$. On this region the free resolvent multiplier $\left(|\xi|^{2}-\lambda_{0}^{2}\right)^{-1}$ is dominated by $|\xi|^{-1}$; hence we see that $R_{0}^{+}\left(\lambda_{0}^{2}\right)(\mu \psi-(\eta * \mu \psi)) \in L^{2}\left(\mathbb{R}^{n}\right)$. This shows that $R_{0}^{+}\left(\lambda_{0}^{2}\right) \mu \psi \in L^{2}\left(\mathbb{R}^{n}\right)$ and hence $\psi \in L^{2}\left(\mathbb{R}^{n}\right)$, which contradicts the assumption that $\lambda_{0}>0$ is not an eigenvalue. Hence $I+R_{0}^{+}\left(\lambda_{0}^{2}\right) \mu$ is invertible.

Remark 3.2. With the stronger assumption $\alpha>n-\frac{2}{n-1}$, one can follow the argument in [11, Proposition 7] to show that $W^{\frac{1}{n+1}, \frac{2 n+2}{n-1}} \subset L^{2}(\mu)$, then apply [16] to conclude that there are no positive eigenvalues of $(-\Delta+\mu)$.

\section{High energy estimates}

The estimates for $R_{0}^{+}\left(\lambda^{2}\right) \mu$ in the preceding sections are adequate for finite intervals of $\lambda$; however the sharp weighted $L^{2}\left(\mathbb{R}^{n}\right)$ resolvent bound from [1] only implies that

$$
\left\|R_{0}^{+}\left(\lambda^{2}\right)\right\|_{\dot{H}^{-1} \rightarrow \dot{H}^{1}} \lesssim 1+|\lambda| .
$$


At high energy one needs to take advantage of the fact that for $f \in L^{2}(\mu), \mu f$ is not a generic element of $\dot{H}^{-1}\left(\mathbb{R}^{n}\right)$. Our main observation at high energy is that the free resolvent in fact has asymptotic decay as an operator on $L^{2}(\mu)$.

Theorem 4.1. Suppose $\mu$ is a compactly supported measure of dimension $\alpha>$ $n-\left(1+\frac{1}{n-1}\right)$. There exists $\varepsilon>0$ so that the free resolvent satisfies

$$
\left\|R_{0}^{+}\left(\lambda^{2}\right) \mu f\right\|_{L^{2}(\mu)} \lesssim\langle\lambda\rangle^{-\varepsilon}\|f\|_{L^{2}(\mu)} .
$$

There are close connections between the free resolvent $R_{0}^{+}\left(\lambda^{2}\right)$ and the restriction of Fourier transforms to the sphere $\lambda \mathbb{S}^{2}$. We make use of a Fourier restriction estimate proved by Du and Zhang [5]. Theorem 2.3 of $[5]$ asserts that for a function $f \in L^{2}\left(\mathbb{R}^{n-1}\right)$ with Fourier support in the unit ball, and a measure $\mu_{R}=R^{\alpha} \mu(\cdot / R)$,

$$
\left\|e^{i t \Delta} f\right\|_{L^{2}\left(\mu_{R}\right)} \lesssim R^{\frac{\alpha}{2 n}}\|f\|_{L^{2}\left(\mathbb{R}^{n-1}\right)}
$$

for sufficiently large $R$. The Schrödinger evolution $e^{i t \Delta} f$ is the inverse Fourier transform (in $\mathbb{R}^{n}$ ) of the measure $\hat{f} \in L^{2}\left(\mathbb{R}^{n-1}\right)$ lifted onto the paraboloid $\Sigma=$ $\left\{\xi_{n}=\left|\xi_{1}\right|^{2}+\cdots+|\xi|_{n-1}^{2}\right\}$. The theorem is then equivalent to the statement

$$
\|(\widehat{g d \Sigma})\|_{L^{2}\left(\mu_{R}\right)} \lesssim R^{\frac{\alpha}{2 n}}\|g\|_{L^{2}(\Sigma)}
$$

for functions $g \in L^{2}(\Sigma \cap B(0,1))$. The use of forward versus inverse Fourier transform does not affect the inequality.

It is well known that the bounded subset of the paraboloid $\Sigma$ can be replaced with any other uniformly convex bounded smooth surface. In this case we wish to apply the result to the unit sphere instead. For any $g \in L^{2}\left(S^{n-1}\right)$,

$$
\|\hat{g}\|_{L^{2}\left(\mu_{R}\right)} \lesssim R^{\frac{\alpha}{2 n}}\|g\|_{L^{2}\left(S^{n-1}\right)} .
$$

The dual statement is

$$
\left\|\left.\widehat{\mu_{R} f}\right|_{|\xi|=1}\right\|_{L^{2}\left(S^{n-1}\right)} \lesssim R^{\frac{\alpha}{2 n}}\|f\|_{L^{2}\left(\mu_{R}\right)} .
$$

Now we reverse some of the scaling relations. Given $f \in L^{2}(\mu)$, let $f_{R}(x)=$ $R^{-\frac{\alpha}{2}} f(x / R)$ so that $\left\|f_{R}\right\|_{L^{2}\left(\mu_{R}\right)}=\|f\|_{L^{2}(\mu)}$. Then $\widehat{\mu_{R} f_{R}}(\xi)=R^{\frac{\alpha}{2}} \widehat{\mu f}(R \xi)$. It follows that

$$
\left\|\left.\widehat{\mu f}\right|_{|\xi|=R}\right\|_{L^{2}\left(R S^{n-1}\right)}=R^{\frac{n-1-\alpha}{2}}\left\|\left.\widehat{\mu_{R} f_{R}}\right|_{|\xi|=1}\right\|_{L^{2}\left(S^{n-1}\right)} \lesssim R^{\frac{n-1}{2}-\frac{\alpha}{2}\left(1-\frac{1}{n}\right)}\|f\|_{L^{2}(\mu)} .
$$

Thanks to the compact support of $\mu$, the $L^{2}(\mu)$ norm of $(1+|x|) f$ is comparable to that of $f$. That allows for control of the derivatives of $\widehat{\mu f}$ with the same restriction bound as in (4.2). In particular we can bound the outward normal gradient of $\widehat{\mu f}$ as

$$
\left\|\left.\frac{\xi}{|\xi|} \cdot \nabla_{\xi}(\widehat{\mu f})(\xi)\right|_{|\xi|=R}\right\|_{L^{2}\left(R S^{n-1}\right)} \lesssim R^{\frac{n-1}{2}-\frac{\alpha}{2}\left(1-\frac{1}{n}\right)}\|f\|_{L^{2}(\mu)} \cdot
$$

Proof of Theorem 4.1. In light of Lemma 3.1] we need only consider $|\lambda| \gtrsim 1$. The specific inequality we derive has the form

$$
\left\|R_{0}^{+}\left(\lambda^{2}\right) \mu f\right\|_{L^{2}(\mu)} \lesssim \lambda^{n-2-\alpha\left(\frac{n-1}{n}\right)} \log \lambda\|f\|_{L^{2}(\mu)} .
$$

Our assumption $\alpha>n-\left(1+\frac{1}{n-1}\right)$ is chosen to make the exponent negative on the right-hand side. 
The free resolvent $R_{0}^{ \pm}\left(\lambda^{2}\right)$ acts by multiplying Fourier transforms pointwise by the distribution

$$
\frac{1}{|\xi|^{2}-\lambda^{2}} \pm i \frac{\pi}{\lambda} d \sigma(|\xi|=|\lambda|)
$$

The surface measure term is $\frac{C}{\lambda}$ times the $T^{*} T$ composition of the operator in (4.2). Thus it has an operator norm bound controlled by $\lambda^{\left[n-1-\alpha\left(\frac{n-1}{n}\right)\right]-1}$.

The multiplier term can be split into two parts depending on whether $|\xi| \sim \lambda$ or not. Let $\phi$ be a smooth function supported in the annulus $\frac{1}{2} \leqslant|\xi|<2$ that is identically 1 when $\frac{3}{4} \leqslant|\xi| \leqslant \frac{3}{2}$. Note that there is an upper bound

$$
\frac{1-\phi(\xi / \lambda)}{|\xi|^{2}-\lambda^{2}} \lesssim \min \left(\lambda^{-2},|\xi|^{-2}\right) \leqslant \frac{1}{\lambda^{\gamma-(n-2)}|\xi|^{n-\gamma}}
$$

for any $n-2 \leqslant \gamma \leqslant n$. Hence this part of the free resolvent maps $\dot{H}^{-\frac{n-\gamma}{2}}\left(\mathbb{R}^{n}\right)$ to $\dot{H}^{\frac{n-\gamma}{2}}\left(\mathbb{R}^{n}\right)$ with an operator norm less than $\lambda^{-(\gamma-(n-2))}$.

The proof of Lemma 2.3 can be modified trivially to show that there is a continuous embedding $j: H^{\frac{n-\gamma}{2}}\left(\mathbb{R}^{n}\right) \mapsto L^{2}(\mu)$ whenever $\gamma<\alpha$ (as required by Lemma 2.2). Since $\alpha>n-2$ this includes a nonempty interval $\gamma \in[n-2, \alpha)$.

By expanding out the free resolvent as $R_{0}^{+}\left(\lambda^{2}\right) \mu=j R_{0}^{+}\left(\lambda^{2}\right) j^{*} \mu$, we see that frequencies $|\xi| \not \lambda$ give rise to an operator on $L^{2}(\mu)$ with norm bound $\lambda^{-(\gamma-(n-2))}$.

The portion of the free resolvent with frequency $|\xi| \sim \lambda$ will be handled by restricting $\widehat{\mu f}$ to spheres of radius $s$, then integrating the results. For each $\frac{\lambda}{2}<s<$ $2 \lambda$ define

$$
F_{s}(x):=\mu f * \widetilde{d \sigma}(\cdot / s)
$$

with $d \sigma$ being the surface measure of the unit sphere. This way, $s^{n-1} \widehat{F}_{s}$ is the restriction of $\widehat{\mu f}$ to the sphere $|\xi|=s$. By the Parseval identity we have an inner product formula for $f, g \in L^{2}(\mu)$,

$$
\left\langle F_{s}, g\right\rangle_{L^{2}(\mu)}=\int_{S^{n-1}} \widehat{\mu f}(s \omega) \overline{\widehat{\mu g}(s \omega)} d \omega=s^{1-n}\langle\widehat{\mu f}, \widehat{\mu g}\rangle_{L^{2}\left(s S^{n-1}\right)} .
$$

Inequality (4.2) shows that $\left\|F_{s}\right\|_{L^{2}(\mu)} \lesssim s^{-\alpha\left(\frac{n-1}{n}\right)}\|f\|_{L^{2}(\mu)}$. If one takes a derivative with respect to $s$, it is easy to apply the product rule to the middle expression. Then the bounds (4.2) and (4.3) show that $\left\|\frac{d}{d s} F_{s}\right\|_{L^{2}(\mu)} \lesssim s^{-\alpha\left(\frac{n-1}{n}\right)}\|f\|_{L^{2}(\mu)}$ as well.

Now the remaining part of the free resolvent appears as a principal value integral

$$
\left\|p . v . \int_{\lambda / 2}^{2 \lambda}\left(\frac{s^{n-1} \phi\left(\frac{s}{\lambda}\right)}{s+\lambda} F_{s}\right) \frac{1}{s-\lambda} d s\right\|_{L^{2}(\mu)} .
$$

Based on the discussion above, both $s^{n-1} \phi\left(\frac{s}{\lambda}\right) F_{s} /(s+\lambda)$ and $\frac{d}{d s}\left[s^{n-1} \phi\left(\frac{s}{\lambda}\right) F_{s} /(s+\right.$ $\lambda)$ are bounded in $L^{2}(\mu)$ with norm less than $\lambda^{n-2-\alpha\left(\frac{n-1}{n}\right)}$ so long as $s \sim \lambda$ and $\lambda \geqslant 4$. The desired bound (4.4) follows by bringing these norms inside the integral when $|s-\lambda|>1$, and integrating by parts once in the more singular interval $|s-\lambda| \leqslant 1$ before bringing the norms inside. The resulting integral in each case is bounded by $\log \lambda$.

We are now able to prove the uniform resolvent bounds in Theorem 1.2 and consequently the Strichartz estimates in Theorem 1.1. 
Proof of Theorem 1.2. The uniform bound (1.10) combines low-energy existence from Corollary 2.6. uniformly on bounded intervals of $\lambda$ from Remark 2.7, and decay as $\lambda \rightarrow \infty$ from Theorem 4.1. The low-energy part of (1.11) is stated as Lemma 3.1. At high energies, we once again apply the resolvent identity (3.2). Theorem 4.1 provides decay of $R_{0}^{+}\left(\lambda^{2}\right) \mu$, and once its norm is less than $\frac{1}{2}$, then the perturbation $\left(I+R_{0}^{+}\left(\lambda^{2}\right) \mu\right)^{-1}$ and consequently $R_{\mu}^{ \pm}\left(\lambda^{2}\right)$ are uniformly bounded as well.

Proof of Theorem 1.1. The derivation of local decay estimates (1.4) and (1.5) for the Schrödinger equation from uniform resolvent bounds follows Kato's argument [14] with minimal adaptation. One can freely interchange the order of the $L_{t}^{2}$ and $L^{2}(\mu)$ norms. Then by a $T T^{*}$ argument, and using the fact that multiplication by $\mu$ is a unitary map between $L^{2}(\mu)$ and its dual space,

$$
\left\|e^{i t \Delta} f\right\|_{L^{2}(\mu) L_{t}^{2}} \leqslant C\|f\|_{2} \quad \text { iff }\left\|\int_{\mathbb{R}} e^{i(t-s) \Delta} \mu g(\cdot, s) d s\right\|_{L^{2}(\mu) L_{t}^{2}} \leqslant C^{2}\|g\|_{L^{2}(\mu) L_{t}^{2}} .
$$

After applying Plancherel's identity to a partial Fourier transform in the time variable, with $\lambda$ as the dual variable to $t$, this is again equivalent (up to a constant) to the bound

$$
\sup _{\lambda \geqslant 0}\left\|\left(R_{0}^{+}(\lambda)-R_{0}^{-}(\lambda)\right) \mu\right\|_{L^{2}(\mu) \rightarrow L^{2}(\mu)} \leqslant C^{2} .
$$

The derivation of (1.5) is identical except the Fourier transform of $e^{i(t-s) H} P_{a c}$ generates the difference of perturbed resolvents $R_{\mu}^{+}(\lambda)-R_{\mu}^{-}(\lambda)$. Negative values of $\lambda$ are excluded because the absolutely continuous spectrum of $-\Delta+\mu$ is still $[0, \infty)$.

The Strichartz inequalities are proved via the argument by Rodnianski and Schlag [19. Use Duhamel's formula to write out the perturbed evolution as

$$
e^{i t(-\Delta+\mu)} P_{a c} f=e^{-i t \Delta} P_{a c} f+i \int_{0}^{t} e^{-i(t-s) \Delta} \mu e^{i s(-\Delta+\mu)} P_{a c} f d s
$$

for $t>0$. Note that $P_{a c}$ is an orthogonal projection, so it is a bounded operator on $L^{2}\left(\mathbb{R}^{n}\right)$. The free evolution term satisfies all Strichartz inequalities including the $p=2$ endpoint. For the inhomogeneous term, our local decay bound (1.5) shows that $\mu e^{i s(-\Delta+\mu)} P_{a c} f \in L_{t}^{2} L^{2}(\mu)^{*}$. The dual statement to (1.4) together with the free Strichartz inequalities imply that

$$
\left\|\int_{\mathbb{R}} e^{-i(t-s) \Delta} G(\cdot, s) d s\right\|_{L_{t}^{p} L_{x}^{q}} \lesssim\|G\|_{L_{t}^{2} L^{2}(\mu)^{*}}
$$

An application of the Christ-Kiselev lemma (for example as stated in 19, Lemma $4.2]$ ) shows that the same bound holds for the desired domain of integration $0 \leqslant$ $s \leqslant t$ provided $p>2$.

\section{REFERENCES}

[1] S. Agmon and L. Hörmander, Asymptotic properties of solutions of differential equations with simple characteristics, J. Analyse Math. 30 (1976), 1-38, DOI 10.1007/BF02786703. MR466902

[2] Horia D. Cornean, Alessandro Michelangeli, and Kenji Yajima, Two-dimensional Schrödinger operators with point interactions: threshold expansions, zero modes and $L^{p}$. boundedness of wave operators, Rev. Math. Phys. 31 (2019), no. 4, 1950012, 32, DOI 10.1142/S0129055X19500120. MR3939663 
[3] Piero D'Ancona, Vittoria Pierfelice, and Alessandro Teta, Dispersive estimate for the Schrödinger equation with point interactions, Math. Methods Appl. Sci. 29 (2006), no. 3, 309-323, DOI 10.1002/mma.682. MR2191432

[4] Gianfausto Dell'Antonio, Alessandro Michelangeli, Raffaele Scandone, and Kenji Yajima, $L^{p}$. boundedness of wave operators for the three-dimensional multi-centre point interaction, Ann. Henri Poincaré 19 (2018), no. 1, 283-322, DOI 10.1007/s00023-017-0628-4. MR 3743762

[5] Xiumin Du and Ruixiang Zhang, Sharp $L^{2}$ estimates of the Schrödinger maximal function in higher dimensions, Ann. of Math. (2) 189 (2019), no. 3, 837-861, DOI 10.4007/annals.2019.189.3.4. MR3961084

[6] M. Burak Erdoğan, Michael Goldberg, and Wilhelm Schlag, Strichartz and smoothing estimates for Schrödinger operators with large magnetic potentials in $\mathbb{R}^{3}$, J. Eur. Math. Soc. (JEMS) 10 (2008), no. 2, 507-531, DOI 10.4171/JEMS/120. MR2390334

[7] M. Burak Erdoğan and William R. Green, Dispersive estimates for the Schrödinger equation for $C^{\frac{n-3}{2}}$ potentials in odd dimensions, Int. Math. Res. Not. IMRN 13 (2010), 2532-2565. MR2669658

[8] J. Ginibre and G. Velo, The global Cauchy problem for the nonlinear Schrödinger equation revisited (English, with French summary), Ann. Inst. H. Poincaré Anal. Non Linéaire 2 (1985), no. 4, 309-327. MR801582

[9] Michael Goldberg, Strichartz estimates for the Schrödinger equation with time-periodic $L^{n / 2}$ potentials, J. Funct. Anal. 256 (2009), no. 3, 718-746, DOI 10.1016/j.jfa.2008.11.005. MR2484934

[10] Michael Goldberg, Dispersive estimates for Schrödinger operators with measure-valued potentials in $\mathbb{R}^{3}$, Indiana Univ. Math. J. 61 (2012), no. 6, 2123-2141, DOI 10.1512/iumj.2012.61.4786. MR.3129105

[11] Michael Goldberg, The Helmholtz equation with $L^{p}$ data and Bochner-Riesz multipliers, Math. Res. Lett. 23 (2016), no. 6, 1665-1679, DOI 10.4310/MRL.2016.v23.n6.a5. MR3621102

[12] Michael Goldberg and Monica Visan, A counterexample to dispersive estimates for Schrödinger operators in higher dimensions, Comm. Math. Phys. 266 (2006), no. 1, 211238, DOI 10.1007/s00220-006-0013-5. MR2231971

[13] J.-L. Journé, A. Soffer, and C. D. Sogge, Decay estimates for Schrödinger operators, Comm. Pure Appl. Math. 44 (1991), no. 5, 573-604, DOI 10.1002/cpa.3160440504. MR.1105875

[14] Tosio Kato, Wave operators and similarity for some non-selfadjoint operators, Math. Ann. 162 (1965/66), 258-279, DOI 10.1007/BF01360915. MR190801

[15] Markus Keel and Terence Tao, Endpoint Strichartz estimates, Amer. J. Math. 120 (1998), no. 5, 955-980. MR 1646048

[16] Herbert Koch and Daniel Tataru, Carleman estimates and absence of embedded eigenvalues, Comm. Math. Phys. 267 (2006), no. 2, 419-449, DOI 10.1007/s00220-006-0060-y. MR2252331

[17] Renato Lucà and Keith M. Rogers, Average decay of the Fourier transform of measures with applications, J. Eur. Math. Soc. (JEMS) 21 (2019), no. 2, 465-506, DOI 10.4171/JEMS/842. MR.3896208

[18] Michael Reed and Barry Simon, Methods of modern mathematical physics. II. Fourier analysis, self-adjointness, Academic Press [Harcourt Brace Jovanovich, Publishers], New YorkLondon, 1975. MR0493420

[19] Igor Rodnianski and Wilhelm Schlag, Time decay for solutions of Schrödinger equations with rough and time-dependent potentials, Invent. Math. 155 (2004), no. 3, 451-513, DOI 10.1007/s00222-003-0325-4. MR2038194

[20] Martin Schechter, Spectra of partial differential operators, North-Holland Series in Applied Mathematics and Mechanics, Vol. 14, North-Holland Publishing Co., Amsterdam-London; American Elsevier Publishing Co., Inc., New York, 1971. MR0447834

[21] Robert S. Strichartz, Restrictions of Fourier transforms to quadratic surfaces and decay of solutions of wave equations, Duke Math. J. 44 (1977), no. 3, 705-714. MR.512086 
Department of Mathematics, University of Illinois, Urbana, Illinois 61801

Email address: berdogan@illinois.edu

Department of Mathematics, University of Cincinnati, Cincinnati, Ohio 45221

Email address: goldbeml@ucmail.uc.edu

Department of Mathematics, Rose-Hulman Institute of Technology, Terre Haute, INDIANA 47803

Email address: green@rose-hulman.edu 